\title{
Material-Specific Recognition Memory Deficits Elicited by Unilateral Hippocampal Electrical Stimulation
}

\author{
Steven G. Coleshill, ${ }^{1,3}$ Colin D. Binnie, ${ }^{1}$ Robin G. Morris, ${ }^{3}$ Gonzalo Alarcón, ${ }^{1}$ Walter van Emde Boas, ${ }^{5}$ \\ Demetrios N. Velis, ${ }^{5}$ Andy Simmons, ${ }^{4}$ Charles E. Polkey, ${ }^{2}$ Cornelis W. M. van Veelen, ${ }^{6}$ and Peter C. van Rijen ${ }^{6}$ \\ Departments of ${ }^{1}$ Clinical Neurophysiology, and ${ }^{2}$ Neurosurgery, King's College Hospital, London, SE5 9RS, United Kingdom, ${ }^{3}$ Department of Psychology \\ and ${ }^{4}$ Neuroimaging Research Group, Institute of Psychiatry, London, SE5 8AF, United Kingdom, ${ }^{5}$ Dutch Epilepsy Clinics Foundation, Heemstede, 2103 SW \\ The Netherlands, and ${ }^{6}$ Department of Neurosurgery, University Medical Center Utrecht, Utrecht, 3508 GA The Netherlands
}

\begin{abstract}
Although the medial temporal lobe is thought to be critical for recognition memory (RM), the specific role of the hippocampus in RM remains uncertain. We investigated the effects of transient unilateral hippocampal electrical stimulation (ES), subthreshold for afterdischarge, on delayed item RM in epilepsy patients implanted with bilateral hippocampal depth electrodes. RM was assessed using a novel computer-controlled test paradigm in which ES to left or right hippocampus was either absent (baseline) or synchronized with item presentation. Subsequent yes-no RM performance revealed a double dissociation between material-specific RM and the lateralization of ES. Left hippocampal ES produced word RM deficits, whereas right hippocampal ES produced face RM deficits. Our findings provide the first demonstration in humans that selective unilateral stimulation-induced hippocampal disruption is sufficient to produce impairments on delayed RM tasks and provide support for the material-specific laterality of hippocampal function with respect to RM.
\end{abstract}

Key words: hippocampus; medial temporal lobe; electrical stimulation; hippocampal depth electrodes; recognition memory; materialspecific effects

\section{Introduction}

The medial temporal lobe (MTL) is unquestionably important for recognition memory (RM). However, the relative contributions of the hippocampus and adjacent entorhinal, perirhinal, and parahippocampal cortices to RM remain to be clearly determined. Early lesion studies in monkeys were marred by extrahippocampal surgical damage. Thus, RM deficits attributed to amygdalo-hippocampal damage were confounded by damage to surrounding tissue, including entorhinal and perirhinal cortex and fibers of passage (Zola-Morgan and Squire, 1986). Subsequently, RM deficits properly attributed to selective hippocampal lesions in monkeys were achieved using radiofrequency or excitotoxic lesions, sparing surrounding tissue and fibers of passage (Alvarez et al., 1995; Beason-Held et al., 1999; Zola et al., 2000). Moreover, selective hippocampal lesions in both humans (ZolaMorgan et al., 1986; Rempel-Clower et al., 1996; Reed and Squire, 1997; Manns and Squire, 1999; Manns et al., 2003; Stark and Squire, 2003) and rats (Mumby et al., 1992, 1995; Wan et al., 1999; Dudchenko et al., 2000; Clark et al., 2000, 2001; Liu and Bilkey, 2001) demonstrably impair RM.

Human subjects with lesions restricted to the hippocampus are rare, but focal unilateral electrical stimulation (ES), sub-

Received Sept. 24, 2003; revised Dec. 12, 2003; accepted Dec. 16, 2003.

This research was supported by grants from the Medical Research Council, the Fund for Epilepsy, and the Epilepsy Research Foundation. This study is dedicated to the memory of Les Law. We are grateful for the assistance given by colleagues: Dzong Nguyen, Tim Cox, Derek Hill, Devi Amin, Beate Gross, Linda Meiners, and Geert Brekelmans.

Correspondence should be addressed to Dr. Steven G. Coleshill, Department of Clinical Neurophysiology, King's College Hospital, Denmark Hill, London, SE5 9RS, UK. E-mail: s.coleshill@iop.kcl.ac.uk.

DOI:10.1523/JNEUROSCI.4352-03.2004

Copyright $\odot 2004$ Society for Neuroscience $\quad$ 0270-6474/04/241612-05\$15.00/0 threshold for afterdischarge (subthreshold ES), offers an alternative unexplored method for investigating effects of selective hippocampal disruption on RM, unconfounded by disruption to other MTL structures. Subthreshold ES acts as a transient lesion, disrupting neural function near the stimulating electrodes while current is applied (Heit et al., 1990), and provides direct insights into the neuropsychological function of stimulated areas by investigating behavioral effects. Previous ES studies by Halgren et al. (1978a,b; Halgren and Wilson, 1985) required afterdischarges (ADs) to disrupt memory performance. Such studies are difficult to interpret, because ADs may propagate widely, preventing localization of ES-induced disruption of neural function. No study, to date, has investigated in humans the effects of selective unilateral hippocampal subthreshold ES on RM. Whereas subthreshold ES-induced visual RM deficits have been demonstrated by stimulating multiple bilateral MTL sites simultaneously (Halgren et al., 1985), this study addressed the specific role of the hippocampus in RM, using a novel computer-controlled RM test paradigm in which focal subthreshold ES to left or right hippocampus was either absent (baseline) or synchronized with presentation of words, faces, or objects during encoding.

\section{Materials and Methods \\ Subjects}

Six right-handed English- or Dutch-speaking patients (four males), with medically intractable complex partial epilepsy, were implanted with intracranial depth electrodes to determine the focus of their seizures for possible surgical resection. Except for one patient with probable mesial temporal sclerosis, all had normal magnetic resonance imaging (MRI). Only patients with bilateral hippocampal depth electrodes that traversed the hippocampus were included. Patients were 19-44 years old (mean 


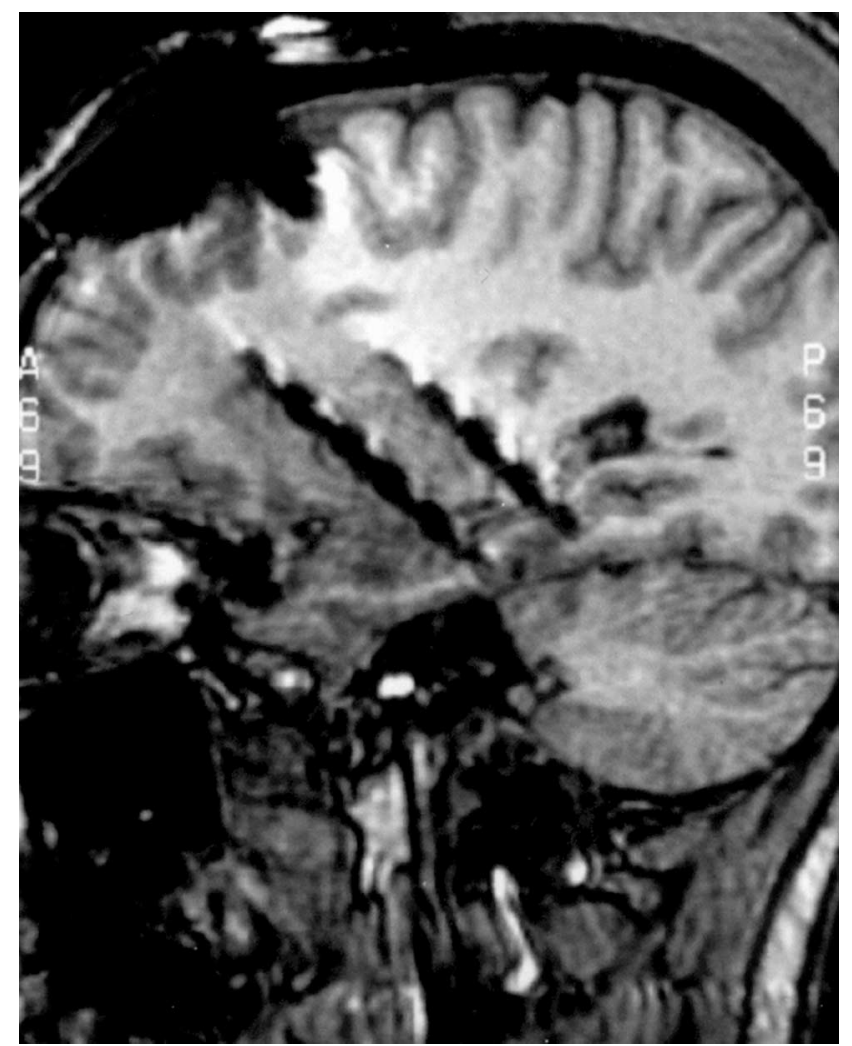

Figure 1. Electrode placement. Postimplantation sagittal MRI showing the trajectory of right anterior and posterior hippocampal depth electrodes. Individual contacts are clearly identified by susceptibility artifact, i.e., signal dropout that appears as black in the image. This is usually larger than the actual size of the electrodes and is most marked at the electrode contacts themselves.

28.5), with mean WAIS-R Full Scale IQ of 98.3, and gave their written informed consent for participation in this ES study, the protocol of which was approved by the Research Ethics Committee of King's College Hospital and was designed in compliance with the clinical intracranial electrostimulation (ICES) protocol of the Dutch Epilepsy Clinics Foundation. The ICES and ES study protocols yielded information regarding seizure lateralization and functional mapping (Velis et al., 1992a,b). However, test results of the ES study did not influence clinical decision making. Antiepileptic medication was reduced during seizure monitoring and partially or totally reinstituted at the time of ICES.

\section{Electrode implantation}

Sites for electrode implantation were based exclusively on clinical criteria. The electrode implantation methodology used has been detailed elsewhere (Agbi and Polkey, 1990; Van Veelen et al., 1990). Up to six, sixcontact nonmagnetic steel or platinum-iridium depth electrode bundles were implanted bilaterally, including anterior and/or posterior hippocampus. Suitable electrode contacts for hippocampal ES were clearly identified by postimplantation MRI using three-dimensional image analysis (Fig. 1).

\section{Stimulation protocol}

The ES protocol remained within the safe parameters suggested by Gordon et al. (1990) for humans. Biphasic constant current ES was delivered in trains of $1 \mathrm{msec}$ pulses at $50 \mathrm{~Hz}$. Maximum charge density was 57 $\mu \mathrm{C} / \mathrm{cm}^{2} /$ pulse at the electrode surface. ES was passed between hippocampal contacts spaced one or two contacts apart using separate OCS-1 Ojemann Cortical Stimulators (Radionics) for each hippocampus. Determination of afterdischarge threshold (ADT) followed established ES protocols (Lesser et al., 1987; Lee et al., 1988, 1990). The memory disruption threshold is just below ADT (Loring et al., 1988; Lee et al., 1990). Thus, ADT was determined separately per hippocampus, and the
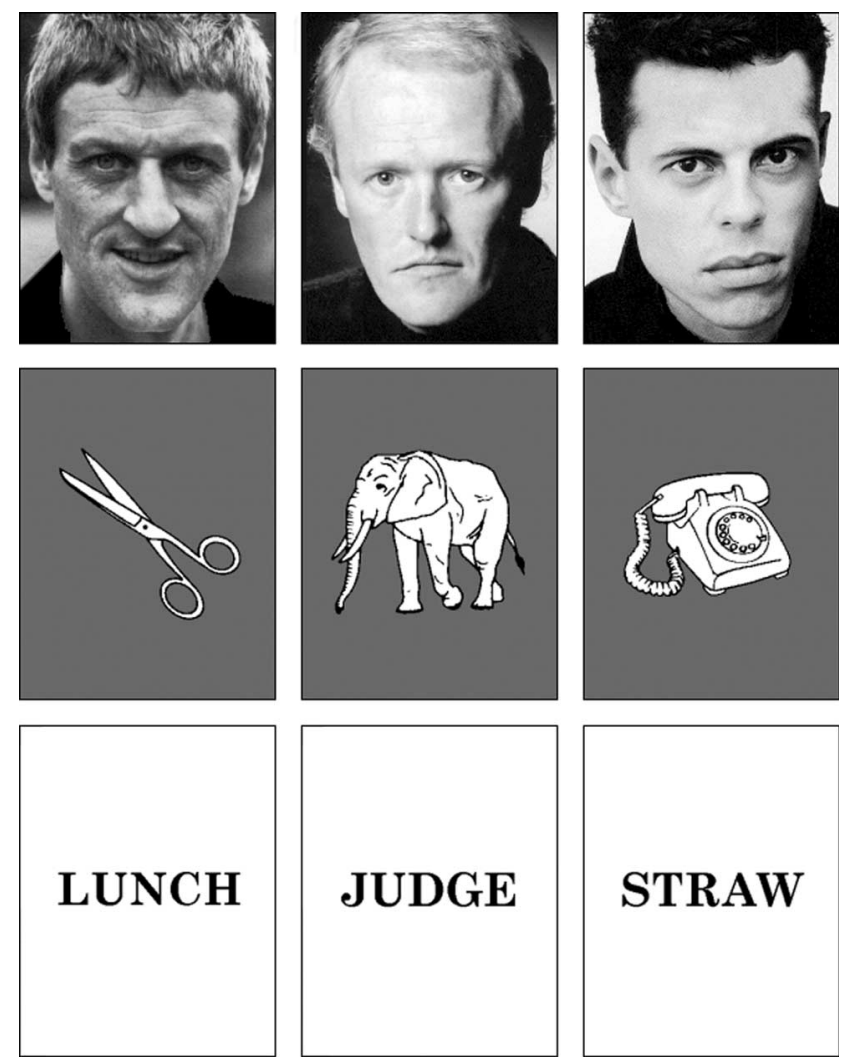

Figure 2. Samples of stimuli presented for separate face, object, and word recognition memory tests.

stimulus strength required to produce functional changes was set at $10-$ $20 \%$ below ADT during test.

\section{Recognition tests}

The computerized RM tests used for this study consisted of a novel yes-no RM paradigm developed by Coleshill (1999), which used trialunique stimuli for two parallel test batteries (RM1 and RM2), each comprising 48 (two parallel blocks of 24) trials per words, faces, and objects test (Fig. 2). Because this study uses a within-subjects methodology, the importance of balancing test items across a range of stimulus attributes (e.g., word frequency) is paramount. This ensures against false-positive ES effects caused by inequalities of stimulus attributes within ES conditions. Thus, test items were rigorously balanced to ensure that rated stimulus attributes were statistically equivalent $(p>0.05)$ for comparisons between the three ES conditions per test.

Words. Ninety-six emotionally neutral four- or five-letter nouns balanced for word frequency: (English and Dutch words: mean, 32.00 and 39.36, respectively); imagery (English words: mean, 5.78; Dutch words: mean, 6.27); age-of-acquisition $(<4.00)$; and familiarity (>3.50) (Gilhooly and Hay, 1977; Gilhooly and Logie, 1980; Van Loon-Vervoorn, 1984; Baayen and Piepenbrock, 1993).

Faces. Ninety-six high-resolution grayscale images of age-matched, unfamiliar white male Caucasian faces, balanced for pose (frontal to threequarters), and rated on seven-point scales for low emotionality (mean, 2.38), and moderate distinctiveness (mean, 4.54) (Coleshill, 1999).

Objects. Ninety-six pictures of black and white line drawings of objects, balanced for name agreement (mean, 86.42) and image agreement (mean, 3.67) (Snodgrass and Vanderwart, 1980).

\section{Design and procedure}

Computer-controlled ES and RM testing was performed while patients were connected to multichannel cable telemetry with CCTV, which registered concurrent display of EEG, onset and offset of ES, RM test stimuli (Fig. 3a), and touch responses from the touchscreen during recognition (Fig. 3b). 
A within-subjects repeated measures design was used where each patient underwent six RM tests: two test batteries (RM1 and RM2) per words, faces, and objects test. RM1 and RM2 data were pooled per subject. Subthreshold tetanic hippocampal ES was time-locked to stimulus presentation $(1.2 \mathrm{sec})$ at encoding. Hence, there were three alternating ES conditions per test: (1) baseline-no ES; (2) ES of left hippocampus; and (3) ES of right hippocampus. Patients were unaware of the onset and offset of ES. In rare cases of localized ADs occurring during RM testing, proceedings were paused for $\sim 30$ min (Lee et al., 1988). Trials with ADs were excluded from analysis.

For each RM test (block 1), subjects were asked to try and remember 12 items (targets), each shown for $1.2 \mathrm{sec}$ on a $43 \mathrm{~cm}$ touchscreen during stimulus presentation. The visual angle subtended by the items was $\sim 12^{\circ}$. A 60 sec distractor interval followed, in which random letters of the alphabet were presented for $450 \mathrm{msec}$ each. Subjects were required to verbalize only the letters " $A$ " or " $B$ " when seen. During the recognition phase, 24 items (12 targets and 12 foils) were presented in pseudorandomized order for $1.2 \mathrm{sec}$ each. After each item, a "yes" or "no" choice on the touchscreen had to be touched within $2.5 \mathrm{sec}$, to indicate whether the item shown was "old" (a target) or "new" (a foil). Targets were shown in reverse serial order, giving a temporal gradient for recognition of 1.15-4.0 min. Then followed block 2. Data were combined across blocks for analysis.

\section{Data analysis}

Choice response time scores and signal detection theory (SDT) parameters for hits $(\mathrm{H})$, misses $(\mathrm{M})$, false alarms $(\mathrm{FA})$, and correct rejections (CR) per RM test were calculated using a computerized SDT analysis program developed by Coleshill (1999). Two measures derived from SDT (MacMillan and Creelman, 1991) were computed on the behavioral data, where $d^{\prime}$ (discrimination $)=z(H)-z(F)$, and $C$ (response bias $)=$ $-0.5[z(H)+z(F)]$. Scores for $d^{\prime}, C$, and choice response time were analyzed using repeated measures multivariate ANOVA (MANOVA) with no between-subjects group factors, but two within-subject factors: three stimulation conditions (baseline; left and right hippocampal ES), and three tests (words, faces, and objects).

\section{Results}

\section{Behavioral measures}

Repeated measures MANOVA on $d^{\prime}$ discrimination scores showed a significant main effect of ES condition, indicating the presence of ES deficits relative to baseline $\left(F_{(2,10)}=5.038 ; p<\right.$ 0.031 ), and a highly significant main effect of test, reflecting the difficulty in recognizing words and faces relative to objects $\left(F_{(2,10)}\right.$ $=20.79 ; p<0.0001$ ) (Fig. 4). A highly significant ES condition $X$ test interaction indicated that $d^{\prime}$ scores varied according to the material-specificity of the test and the lateralization of hippocampal ES $\left(F_{(4,20)}=5.008 ; p<0.006\right)$. Subsequent investigation of these effects were made using paired $t$ tests as contrasts. Protection against the type I error using multiple comparisons was ensured by using a more stringent significance level of $p<0.01$. Compared with baseline, left hippocampal ES (LH-Stim) induced significant RM deficits for words $\left(t_{(5)}=3.85 ; p<0.01\right.$ ), whereas right hippocampal ES (RH-Stim) induced significant RM deficits for faces $\left(t_{(5)}=4.29 ; p<0.008\right)$. These data represent a double dissociation between ES and test, such that material-specific RM is dependent on the lateralization of hippocampal ES. Left hippocampal ES impaired word RM, whereas right hippocampal ES impaired face RM. Results for objects were not significant.
If there is no response bias, then $C=0$. $C$ response bias scores show little deviation from neutral bias (Table 1). Similarly, choice response time is restricted to a range of $0.46-0.60 \mathrm{sec}$. There were no significant effects for response bias or choice response time for any of the RM tests.

\section{Discussion}

These experimental findings provide, to our knowledge, the first demonstration in humans that transient unilateral subthreshold ES-induced hippocampal disruption during stimulus encoding is sufficient to produce impairment on subsequent delayed yes-no RM. Moreover, the pattern of impairment revealed a classic dou-

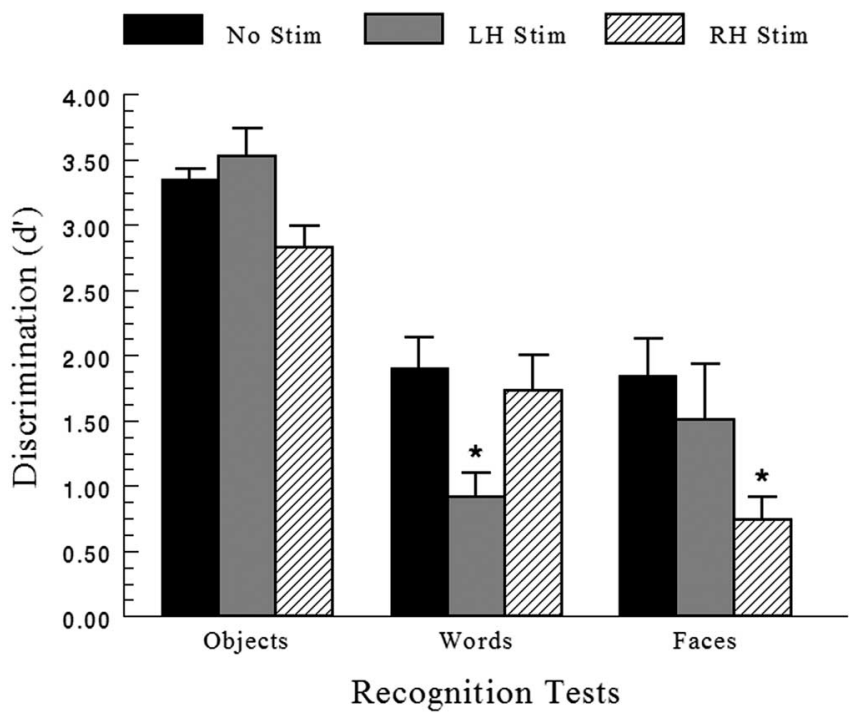

Figure 4. Material-specific delayed RM scores ( $d^{\prime}$ discrimination) (+ 1 SEM) for left (LHStim) and right (RH-Stim) hippocampal ES compared with unstimulated baseline (No Stim) for each RM test. Zero on the ordinate represents chance performance. ${ }^{*} p<0.01$. 
Table 1. C response bias scores and choice response time (Choice RT) (sec) for each RM test, for unstimulated baseline and left (LH-Stim) and right (RH-Stim) hippocampal stimulation conditions

\begin{tabular}{|c|c|c|c|c|c|c|}
\hline & \multicolumn{2}{|l|}{ Objects } & \multicolumn{2}{|l|}{ Words } & \multicolumn{2}{|l|}{ Faces } \\
\hline & Bias & Choice RT & Bias & Choice RT & Bias & Choice RT \\
\hline Baseline & $0.09 \pm 0.18$ & $0.53 \pm 0.11$ & $-0.27 \pm 0.23$ & $0.55 \pm 0.10$ & $-0.01 \pm 0.27$ & $0.53 \pm 0.05$ \\
\hline LH-Stim & $-0.01 \pm 0.19$ & $0.53 \pm 0.12$ & $0.07 \pm 0.14$ & $0.60 \pm 0.10$ & $0.16 \pm 0.25$ & $0.46 \pm 0.06$ \\
\hline RH-Stim & $0.05 \pm 0.22$ & $0.54 \pm 0.09$ & $-0.02 \pm 0.29$ & $0.56 \pm 0.10$ & $0.13 \pm 0.26$ & $0.50 \pm 0.05$ \\
\hline
\end{tabular}

Mean \pm SEM. C scores less than zero represent liberal "yes" bias, and C scores more than zero represent conservative "no" bias. pal amnesic patients are equally well known (Milner, 1966). Similarly, delaydependent RM deficits have been reported after hippocampal damage (Alvarez et al., 1995; Buffalo et al., 1998; Dudchenko et al., 2000). In contrast to the RMT, which has no distractor interval, we used RM tests containing a $1 \mathrm{~min}$ interpolated distractor task to prevent rehearsal, with a temporal gradient for recognition of a fur-

ble dissociation between material-specific RM and the lateralization of ES. Left hippocampal ES impaired word RM, whereas right hippocampal ES impaired face RM (Fig. 4). The present findings thus provide direct evidence for a functional role of the hippocampus in delayed RM and concur with recent studies in humans (Zola-Morgan et al., 1986; Rempel-Clower et al., 1996; Reed and Squire, 1997; Manns and Squire, 1999; Manns et al., 2003; Stark and Squire, 2003), monkeys (Alvarez et al., 1995; Beason-Held et al., 1999; Zola et al., 2000), and rats (Mumby et al., 1992, 1995; Wan et al., 1999; Dudchenko et al., 2000; Clark et al., 2000, 2001; Liu and Bilkey, 2001), which have shown that selective hippocampal lesions impair RM.

To our knowledge, no previous study has investigated the effects of unilateral hippocampal ES on delayed RM, so no material-specific effects are comparable. Moreover, the temporal lobe amnesia-based RM literature is inapplicable, which frequently includes subjects with selective hippocampal but predominantly bilateral lesions (Zola-Morgan et al., 1986; RempelClower et al., 1996). However, our findings converge with multidisciplinary studies that have shown the involvement of the left hippocampus in word-memory (Lee et al., 1988, 1990; Stark and Squire, 2001), and the right hippocampus in face-memory (Grady et al., 1995; Haxby et al., 1996). ES-induced effects on RM for objects were absent. Because patients found the objects test easy, this result can be explained parsimoniously by ceiling effects. Another possibility is that RM for objects requires dualencoding (visual and verbal) (Simons et al., 2001), so that bilateral ES would probably be necessary to show an effect. ESinduced RM disruption was clearly not the result of a confusional state or impaired attentional processing, because subthreshold ES was used. The lack of an effect for objects confirms this.

Several aspects of the RM test paradigm may have contributed to the magnitude of RM deficits reported here. First, yes-no RM tests, in which target and foil items are shown separately, are more cognitively demanding by a factor of the square root of 2 , than two-alternative forced choice (2AFC) tests of RM, in which target and foil items are shown together (MacMillan and Creelman, 1991). Thus, 2AFC tests of RM such as the Warrington Recognition Memory Test (RMT) in humans (Warrington, 1984), and delayed nonmatching-to-sample tasks in nonhuman animals, may be less likely to involve the hippocampus as a function of reduced cognitive demand. Our test paradigm emphasized hippocampally dependent recollection-based processes during encoding (Schacter et al., 1996), because subjects were explicitly asked to remember items. For the words test, subjects were asked to remember the "meaning" of words shown (deep encoding); a process thought to be left hippocampally-dependent (Otten et al., 2001).

Second, tests involving delays and distractions are known to be acutely sensitive to hippocampal damage in humans and monkeys (Zola-Morgan and Squire, 1985; Alvarez et al., 1995; Clark et al., 2001). Rapid forgetting in the absence of continuous verbal rehearsal and the catastrophic effects of distraction in hippocam- ther $3 \mathrm{~min}$. Thus, we conclude that the susceptibility of the hippocampus to the effects of ES-induced disruption at encoding in this study may have been augmented both by the cognitive demand and recollective aspects of the yes-no RM tests, with subsequent weakening of the putative memory trace by the combined effects of distraction and delay.

Neuroimaging studies of RM have shown hippocampal involvement during encoding only (Grady et al., 1995; Haxby et al., 1996), subsequent retrieval (Stark and Squire, 2000), or both (Gabrielli et al., 1997). Such reports of robust hippocampal activation during encoding are congruent with the ES study by Halgren et al. (1985), which showed that RM is more susceptible to MTL disruption at encoding than during subsequent retrieval, although both processes were clearly disrupted. Our results indicate that hippocampal ES, time-locked to stimulus encoding, disrupted the neural substrate for discriminating novel from repeated stimuli by interfering with memory trace formation. The $600 \mathrm{msec}$ immediately after stimulus presentation has been shown to be the critical period during which MTL synaptic activity contributes to processes of associative stimulus integration during human memory encoding (Heit et al., 1990). Similarly, subthreshold 20-50 msec ES of the MTL, delivered within 200 msec of stimulus presentation, was found to be maximally disruptive of subsequent RM in monkeys (Ringo, 1995). Taskirrelevant action potentials during ES are thought to disrupt memory during stimulus encoding (Ringo, 1995), consistent with the observation that multiple-unit activity during subthreshold 100 msec single-pulse ES showed a sequence of synchronous excitation and inhibition of hippocampal neurons lasting for $\sim 400 \mathrm{msec}$ (Halgren et al., 1985).

In conclusion, this stimulation study demonstrates the efficacy of unilateral hippocampal ES in eliciting material-specific RM deficits. Our findings show that computer-controlled transient focal unilateral subthreshold ES is an effective paradigm for investigating the functional equivalent of selective surgical hippocampal lesions on memory function (Alvarez et al., 1995; Murray and Mishkin, 1998; Beason-Held et al., 1999; Zola et al., 2000), which is unconfounded by disruption to other structures within the MTL. Historically, bilateral ES of the MTL has proved useful as a model of amnesia (Halgren et al., 1978a,b; Halgren and Wilson, 1985). In contrast, focal unilateral hippocampal subthreshold ES permits the structure-function relationships of left and right hippocampus to be evaluated independently, and for material-specific effects to be systematically explored, unconfounded by possible global amnesic effects of bilateral ES.

\section{References}

Agbi C, Polkey CE (1990) Calculation of coordinates for depth electrodes placed in temporal lobe structures visualized by oblique CT scan cuts. Br J Neurosurg 4:517-521.

Alvarez P, Zola-Morgan S, Squire LR (1995) Damage limited to the hippocampal region produces long-lasting memory impairment in monkeys. J Neurosci 15:3796-3807.

Baayen RH, Piepenbrock R (1993) The CELEX lexical database (CD-ROM). 
Linguistic Data Consortium, University of Pennsylvania, Philadelphia, PA.

Beason-Held IL, Rosene DL, Killiany RJ, Moss MB (1999) Hippocampal formation lesions produce memory impairment in the rhesus monkey. Hippocampus 9:562-574.

Buffalo EA, Reber PJ, Squire LR (1998) The human perirhinal cortex and recognition memory. Hippocampus 8:330-339.

Clark RE, Zola SM, Squire LR (2000) Impaired recognition memory in rats after damage to the hippocampus. J Neurosci 20:8853-8860.

Clark RE, West AN, Zola SM, Squire LR (2001) Rats with lesions of the hippocampus are impaired on the delayed nonmatching-to-sample task. Hippocampus 11:176-186.

Coleshill SG (1999) Neuropsychological correlates of unilateral mesial temporal sclerosis and alternate unilateral subthreshold electrical stimulation of the hippocampus. Unpublished PhD thesis, London University: London, UK.

Dudchenko PA, Wood ER, Eichenbaum H (2000) Neurotoxic hippocampal lesions have no effect on odor span and little effect on odor recognition memory but produce significant impairments on spatial span, recognition, and alternation. J Neurosci 20:2964-2977.

Gabrielli JDE, Brewer JB, Desmond JE, Glover GH (1997) Separate neural bases of two fundamental memory processes in the human medial temporal lobe. Science 276:264-266.

Gilhooly KJ, Hay D (1977) Imagery, concreteness, age of acquisition, familiarity, and meaningfulness values for 205 five-letter words having singlesolution anagrams. Behav Res Meth Instr 9:12-17.

Gilhooly KJ, Logie RH (1980) Age of acquisition, imagery, concreteness, familiarity, and ambiguity measures for 1, 944 words. Behav Res Meth Instr 12:395-427.

Gordon B, Lesser RP, Rance NE, Hart Jr J, Webber R, Uematsu S, Fisher RS (1990) Parameters for direct cortical electrical stimulation in the human: histopathologic conformation. Electr Clin Neurophysiol 75:371-377.

Grady CL, McIntosh AR, Horwitz B, Maisog JM, Ungerleider LG, Mentis MJ, Pietrini P, Schapiro MB, Haxby JV (1995) Age-related reductions in human recognition memory due to impaired encoding. Science 269:218-220.

Halgren E, Wilson CL (1985) Recall deficits produced by afterdischarges in the human hippocampal formation and amygdala. Electr Clin Neurophysiol 61:375-380.

Halgren E, Babb TL, Crandall PH (1978a) Activity of human hippocampal formation and amygdala neurons during memory testing. Electr Clin Neurophysiol 45:585-601.

Halgren E, Walter RD, Cherlow DG, Crandall H (1978b) Mental phenomena evoked by electrical stimulation of the human hippocampal formation and amygdala. Brain 101:83-117.

Halgren E, Wilson CL, Stapleton J (1985) Human medial temporal-lobe disruption disrupts both formation and retrieval of recent memories. Brain Cog 4:287-295.

Haxby JV, Ungerleider LG, Horowitz B, Maisog JM, Rapoport SI, Grady CL (1996) Face encoding and recognition in the human brain. Proc Natl Acad Sci USA 93:922-927.

Heit G, Smith ME, Halgren E (1990) Neuronal activity in the human medial temporal lobe during recognition memory. Brain 113:1093-1112.

Lee GP, Loring DW, Flanigan HF, Smith JR, Meador K (1988) Electrical stimulation of the human hippocampus produces verbal intrusions during memory testing. Neuropsychology 26:623-627.

Lee GP, Loring DW, Smith JR, Flanigin HF (1990) Material specific learning during electrical stimulation of the human hippocampus. Cortex 26:433-442.

Lesser RP, Lüders H, Klem G, Dinner DS, Morris HH, Hahn JF, Wyllie E (1987) Extraoperative cortical functional localization in patients with epilepsy. J Clin Neurophysiol 4:27-53.

Liu P, Bilkey DK (2001) The effect of excitotoxic lesions centered on the hippocampus or perirhinal cortex in object recognition and spatial memory tasks. Behav Neurosci 115:94-111.

Loring DW, Lee GP, Flanigan HF, Meador KJ, Smith JR, Gallagher BB, King DW (1988) Verbal memory performance following unilateral electrical stimulation of the human hippocampus. J Epilepsy 1:79-85.

MacMillan NA, Creelman CD (1991) Detection theory: a user's guide. Cambridge, Cambridge UP.

Manns JR, Squire LR (1999) Impaired recognition memory on the doors and people test after damage limited to the hippocampal region. Hippocampus 9:495-499.
Manns JR, Hopkins RO, Reed JM, Kitchener EG, Squire LR (2003) Recognition memory and the human hippocampus. Neuron 37:171-180.

Milner B (1966) Amnesia following operation on the temporal lobes. In: Amnesia (Whitty CWM, Zangwill OL, eds), pp 109-133. London: Butterworths.

Mumby DG, Pinel JPJ, Kornecook TJ (1992) Dissociating the effects of hippocampal and amygdalar lesions in rats with a battery of nonspatial memory tasks. Soc Neurosci Abstr 18:1423.

Mumby DG, Pinel JPJ, Kornecook TJ, Shen MJ, Redila VA (1995) Memory deficits following lesions of hippocampus or amygdala in rat: assessment by an object-memory test battery. Psychobiology 23:26-36.

Murray EA, Mishkin M (1998) Object recognition and location memory in monkeys with excitotoxic lesions of the amygdala and hippocampus. J Neurosci 18:6568-6582.

Otten LJ, Henson RN, Rugg MD (2001) Depth of processing effects on neural correlates of memory encoding: relationship between findings from across- and within-task comparisons. Brain 124:399-412.

Reed JM, Squire LR (1997) Impaired recognition memory in patients with lesions limited to the hippocampal formation. Behav Neurosci 111:667-675.

Rempel-Clower NL, Zola-Morgan S, Squire LR, Amaral DG (1996) Three cases of enduring memory impairment after bilateral damage limited to the hippocampal formation. J Neurosci 16:5233-5255.

Ringo JL (1995) Brevity of processing in a mnemonic task. J Neurophysiol 73:1712-1715.

Schacter DL, Alpert NM, Savage CR, Rauch SL, Albert MS (1996) Conscious recollection and the human hippocampal formation: evidence from positron emission tomography. Proc Natl Acad Sci USA 93:321-325.

Simons JS, Graham KS, Owen AM, Patterson K, Hodges JR (2001) Perceptual and semantic components of memory for objects and faces: a PET study. J Cogn Neurosci 13:430-443.

Snodgrass JG, Vanderwart M (1980) A standardized set of 260 pictures: norms for name agreement, image agreement, familiarity, and visual complexity. J Exp Psychol Hum Learn Mem 6:174-215.

Stark CE, Squire LR (2000) Functional magnetic resonance imaging (fMRI) activity in the hippocampal region during recognition memory. J Neurosci 20:7776-7781.

Stark CE, Squire LR (2001) Simple and associative recognition memory in the hippocampal region. Learn Mem 8:190-197.

Stark CE, Squire LR (2003) Hippocampal damage equally impairs memory for single items and memory for conjunctions. Hippocampus 13:281-292.

Van Loon-Vervoorn WA (1984) Afbeeldbaarheidsnormen van 1087 Nederlandse woorden. Bijlage bij artikel in het Nederlands Tijdschrift voor de Psychologie, 1-32.

Van Veelen CWM, Debets RMC, Van Huffelen AC, Van Emde Boas W, Binnie CD, Storm Van Leeuwen W, Velis DN, Van Dieren A (1990) Combined use of subdural and intracerebral electrodes in preoperative evaluation of epilepsy. J Neurosurg 26:93-101.

Velis DN, Brekelmans G, Van Emde Boas W (1992a) Intracranial electrostimulation (ICES) and functional mapping (FM) in temporal lobe epilepsy (TLE). Electroencephalogr Clin Neurophysiol 82:94P.

Velis DN, Brekelmans GJF, Van Emde Boas W, De Vries J, Van Egmond P, Klepper J, Dekker E, Van Veelen CWM (1992b) Lateralizing value of electrical stimulation of the hippocampus in temporal lobe epilepsy. Epilepsia 33 [Suppl 3]:87.

Wan H, Aggleton JP, Brown MW (1999) Different contributions of the hippocampus and perirhinal cortex to recognition memory. J Neurosci 19: 3:1142-1148.

Warrington EK (1984) Recognition memory test manual. NFER-Nelson, Windsor.

Zola-Morgan S, Squire LR (1985) Medial temporal lesions in monkeys impair memory on a variety of tasks sensitive to human amnesia. Behav Neurosci 99:22-34.

Zola-Morgan S, Squire LR (1986) Memory impairment in monkeys following lesions limited to the hippocampus. Behav Neurosci 100:155-160.

Zola-Morgan S, Squire LR, Amaral DG (1986) Human amnesia and the medial temporal region: Enduring memory impairment following a bilateral lesion limited to the CA1 field of the hippocampus. J Neurosci 6:2950-2967.

Zola SM, Squire LR, Teng E, Stefanicci L, Buffalo EA, Clark RE (2000) Impaired recognition memory in monkeys after damage limited to the hippocampal region. J Neurosci 20:451-463. 